\section{A cluster of Pneumocystis carinii infections in children}

Pneumocystis carinii pneumonia occurs almost exclusively in immunosuppressed patients, particularly those being treated for acute lymphoblastic leukaemia. The mode of transmission of the organism is not clear. ${ }^{1}$ Several hospital outbreaks have been reported, and animal to animal transmission has been documented, but convincing evidence is still lacking of person to person transmission. We present a cluster of children currently receiving the United Kingdom Acute Lymphoblastic Leukaemia eight (UKALL 8) regimen for acute lymphoblastic leukaemia who became seropositive for antipneumocystis antibody within a short space of time, providing further evidence for person to person transmission.

\section{Patients, methods, and results}

Children currently receiving the UKALL 8 regimen for acute lymphoblastic leukaemia have an indirect immunofluorescence test performed monthly by Coon's technique ${ }^{2}$ for the detection of IgG antibodies to $P$ carinii. Six children in a paediatric ward aged $31 / 2$ to 8 years were being so treated, and between Novembe 1983 and May 1984 five of them (who were all previously seronegative) developed antibodies in significant titre to $P$ carinii. Only two of the children developed a pneumonitis clinically and radiologically resembling $P$ carinii infection. Both were admitted and responded promptly to co-trimoxazole. Invasive diagnostic procedures were not performed.

On reviewing retrospectively the remaining three seropositive children we noted that all three had symptoms of upper respiratory tract infection with fever and a dry cough coinciding with the times of rising antibody titre.

One of the children was found to have an extremely high titre of antibodies. She was the only patient in whom IgM antibodies to $P$ carinii were measured, and this titre was also raised. All six children attended an outpatient department simultaneously once a week, spending about an hour together in a small waiting room. All had been admitted to hospital at various stages but never at the same time. No patient had been given prophylactic co-trimoxazole during the previous five to six months. When last measured all the titres were falling.

The figure shows the titres and times of increase of antibodies in the five patients.

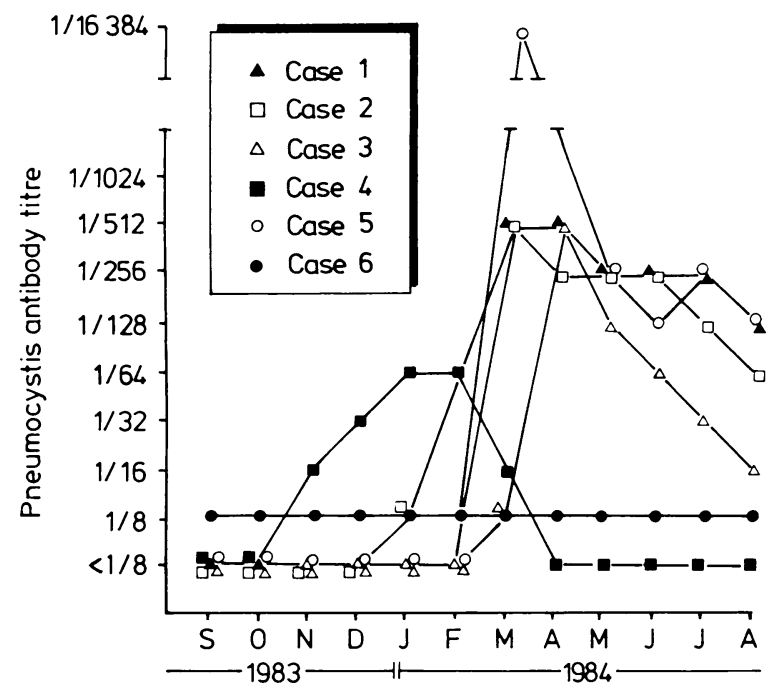

Relation of rising pneumocystis antibody titres with respect to time.

\section{Comment}

Several workers have found antibodies to $P$ carinii in three quarters to all of the population studied by the age of 4 years. ${ }^{34}$ This suggests universal exposure and subclinical infection with $P$ carinii, the disease becoming evident clinically only in immunosuppressed patients.

There have been four major outbreaks of Pneumocystis pneumonia reported in patients with malignant disease, all of which occurred in North America. 'Two of these were considered to be due to reactivation, probably secondary to highly intensive chemotherapy regimens. In the remaining two, person to person spread was thought to be a possibility.

In our series also cross infection among the patients would be the most likely explanation, for several reasons. Firstly, all five patients had negative titres initially, implying that previous exposure had not occurred (although it may indicate suppression of antibodies ${ }^{2}$ from chemotherapy). The remaining child already had a low titre of antibodies to $P$ carinii and thus may have been protected. Secondly, the rise in titre in all patients was seen over six months. An interval of four to eight weeks occurred between cases, consistent with the incubation period of $P$ carinii infection. Thirdly, the only patient tested for IgM specific $P$ carinii antibodies had these in high titre, indicating a primary infection.

An alternative but less likely explanation for our findings would be that some common factor might have induced reactivation of latent infection.

We thank Dr B Jameson and Mrs J Skilton, Royal Marsden Hospital, for their help. We are grateful to Mrs Mary Foody for secretarial work.

1 Hughes WT, Bartley DL, Smith BM. A natural source of infection due to Pneumocystis carinii J Infect Dis 1982;145:842-8

Shepherd V, Jameson B, Knowles GK. Pneumocystis carinii pneumonitis: a serological study. f Clin Pathol 1979;32:773-7.

Meuwissen JN'TT, Tauber I, Leeuwenberg ADEM, Berkers PJA, Sieben M. Parasitologic and serologic observations of infection. I Infect Dis 1977;136:43-9.

4 Pifer LL, Hughes WT, Stagno S, Woods D. Pneumocystis carinii infection: evidence for high prevalence in normal and immunosuppressed children. Pediatrics 1978;61:35-41.

5 Rhame FS, Streifel AJ, Kersey JM, McGlave PB. Extrinsic risk factors for pneumonia in the patient at high risk of 'infection.' Am $\mathcal{F}$ Med 1984;76:5A, 49-52.

(Accepted 18 September 1985)

Department of Clinical Microbiology (Trinity College), St James's Hospital, Dublin 8

LYNDA E FENELON, MB, MRCPI, microbiology registrar

CONOR T KEANE, MB, FRCPATH, consultant microbiologist

National Children's Hospital, Dublin 2

MAHMOUD BAKIR, MB, MRCP, paediatric registrar

IAN J TEMPERLEY, MD, FRCPATH, consultant haematologist

Correspondence to: Dr Fenelon.

\section{Local anaesthetic infiltration versus Bier's block for Colles' fractures}

After recent concern' about the safety of Bier's intravenous regional anaesthesia for manipulation of Colles' fractures in outpatients, ${ }^{2}$ we have considered the simpler and quicker method of local anaesthetic infiltration directly into the haematoma of the fracture. A series of 280 patients treated with this method showed good tolerance and absence of pain for 4-24 hours after manipulation.

Objections to local infiltration are the possibility of infection, a more painful injection, and difficult reduction with less relaxation of muscles. A prospective randomised trial was carried out to test the effectiveness and duration of each treatment and note complications or side effects.

\section{Patients, methods, and results}

One hundred consecutive patients requiring manipulation of Colles' fractures and satisfying the following criteria were randomly allocated to receive either Bier's block or local infiltration: aged over 15; normal sensation and circulation of limb; closed injury; no diabetes, epilepsy, or sickle cell disease; and no relevant allergy.

The patient's forearm was prepared with $2 \%$ chlorhexidine in spirit and draped with sterile towels. Wearing sterile gloves, the operator injected $10 \mathrm{ml}$ of $2 \%$ lignocaine into the cavity of the fracture and around the adjacent periosteum, including the ulnar styloid. The arm was raised for one minute before inflating the upper part of a double cuff to $250 \mathrm{~mm} \mathrm{Hg}$; then $30-40 \mathrm{ml}$ of $0.5 \%$ prilocaine was injected into a vein on the back of the hand. The lower cuff was inflated and the upper one deflated 20 minutes later. The fracture was manipulated 10 minutes after injection in each group.

Pain scores were recorded on $10 \mathrm{~cm}$ linear analogue scales ${ }^{4}$ representing the range from "no pain" to "very severe pain" before beginning the procedure, during injection of local anaesthetic, during manipulation, and when plaster was completed during radiography. The casualty officer recorded his impression of the effectiveness of the technique on a linear analogue scale and noted any difficulties. The times of first consultation, start of procedure, completion of plaster, and release of tourniquet were recorded.

Records of 83 patients were completed adequately and analysed. The groups were comparable in age, sex, dominance of affected wrist, and degree of displacement of fracture. Forty four received Bier's block. Pain scores during manipulation were higher for patients receiving local infiltration (mean 5.53 (SD $3.67)$ ) than for those receiving Bier's block $(2.64(3.02))(p<0.0003$ using Student's $t$ test). At other stages the scores were not significantly different between groups. The casualty officer's impression of effectiveness was usually more generous than the patient's but showed the same superiority of Bier's block $(7 \cdot 68(2 \cdot 38))$ over local infiltration $(6 \cdot 11(2 \cdot 84))(\mathrm{p}<0 \cdot 009)$.

No difference was noticed in the period of postoperative painlessness between the groups: Bier's block $3 \cdot 7(3.0)$ hours; local infiltration $4 \cdot 0(3 \cdot 0)$ hours. The 